\title{
Tuberculose au Canada - La bataille se poursuit
}

\author{
Alvarez $\mathrm{GG}^{1,23^{*}}$
}

\author{
${ }^{1}$ Institut de recherche de l'Hôpital d'Ottawa, Université d'Ottawa, Ottawa (Ontario) \\ 2 Université d'Ottawa, Faculté de médecine, Ottawa (Ontario) \\ ${ }^{3}$ L'Hôpital d'Ottawa, Départment de médecine, Division de pneumologie et des maladies infectieuses, Ottawa (Ontario), \\ Canada
}

*Correspondance : galvarez@toh.on.ca

Dans cette édition du Relevé des maladies transmissibles au Canada, Gallant et al. présentent les données de 2013 du Système canadien de déclaration des cas de tuberculose, lesquelles semblent indiquer que le taux d'incidence de la tuberculose active au Canada n'a pas changé par rapport à 2012 et est stable depuis 2007, soit moins de 5 cas par 100000 habitants (1). Bien qu'on puisse se réjouir du fait que le taux de tuberculose soit faible au Canada depuis de nombreuses années, il faut souligner qu'il est possible d'en faire plus pour le réduire encore davantage. Le taux d'incidence de tuberculose est plus élevé chez les Autochtones nés au Canada (19,9 cas pour 100000 habitants) que dans tous les autres groupes, y compris les personnes nées à l'étranger (14,8 cas pour 100000 habitants). Toutefois, en termes absolus, le fardeau de la maladie est plus élevé chez les personnes nées à l'étranger, soit 1146 cas contre 309 cases (1). L'article souligne également que les Autochtones nés au Canada représentent $4 \%$ de la population canadienne, mais $19 \%$ de tous les cas de tuberculose active (1).

Le taux d'incidence le plus élevé au Canada a été observé au Nunavut, à savoir 143 cas pour 100000 habitants en 2013 (1). Le taux d'incidence de tuberculose active au Nunavut montre une tendance à la hausse depuis 10 ans et a atteint 304 cas pour 100000 habitants en 2010, ce qui représente 101 cas actifs. Le Nunavut fait partie de la région appelée Inuit Nunangat (terre des Inuits), qui englobe quatre régions de l'Arctique canadien. Les Inuits représentent $85 \%$ de la population du Nunavut. Ils présentent un taux de tuberculose anormalement élevé au regard du reste des autres Autochtones nés au Canada. Dans l'article de Gallant et al. qui fait l'objet de la présente édition, les taux d'incidence signalés pour les Autochtones des Premières nations et les Métis nés au Canada étaient respectivement de 21,8 cas pour 100000 habitants et de 3,3 cas pour 100000 habitants, tandis que le taux d'incidence chez les Inuits (dans l'ensemble de l'Inuit Nunangat) était de 154,2 pour 100000 habitants, soit plus de 30 fois le taux d'incidence canadien (1). Fait à noter, même si les Inuits présentent le taux le plus élevé, c'est chez les Autochtones des Premières nations que le fardeau de la maladie est le plus lourd au Canada parmi les Autochtones nés au Canada. Par ailleurs, le taux d'incidence déclaré pour les Premières nations est stable depuis plus de dix ans, alors que le taux chez les Canadiens non autochtones n'a cessé de diminuer. On a récemment observé que la distribution géographique de la tuberculose chez les Premières nations était caractérisée par une concentration très élevée dans le nord des Prairies et que le taux d'incidence augmentait d'ouest en est (2).

Ces taux élevés de tuberculose parmi les Autochtones du Canada se maintiennent malgré les programmes relatifs à la santé publique qui sont consacrés à la prévention et au contrôle de la tuberculose et qui répondent aux Normes canadiennes pour la lutte antituberculeuse (3). La prévention et le contrôle de la tuberculose chez les Autochtones nés au Canada présentent des difficultés particulières, notamment la dispersion géographique sur de vastes territoires, la complexité de la prestation des soins de santé, la nécessité d'offrir des soins appropriés sur le plan culturel, les facteurs de risque et la prévalence de déterminants sociaux de la santé qui ne sont pas les mêmes d'un endroit à l'autre en ce qui a trait à la tuberculose (4).

Toute approche réussie en matière de prévention et de contrôle de la tuberculose chez les Autochtones doit impérativement faire appel aux Autochtones eux-mêmes quant à la recherche de solutions. Les solutions de proximité sont souvent les mieux ciblées et les plus viables. L'organisation inuite nationale Inuit Tapiriit Kanatami (ITK) a mis sur pied un groupe de travail stratégique pour la lutte contre la tuberculose chez les Inuits, auquel participent des organismes gouvernementaux fédéraux, provinciaux et territoriaux (5). La stratégie de lutte contre 
la tuberculose chez les Inuits comporte un cadre et un processus visant à aider les partenaires à élaborer un plan d'action propre aux Inuits, qui comprend notamment des éléments clés sur le plan de l'éducation et de la mobilisation communautaires, des partenariats visant à améliorer les déterminants sociaux de la santé ainsi que de nouvelles études portant sur la prévention et le contrôle de la tuberculose (5). Le groupe de travail énonce cinq éléments centraux du plan d'action en soulignant de nombreux obstacles auxquels font face les Inuits dans ces régions en ce qui concerne la prévention et le contrôle de la tuberculose. Santé Canada et un comité d'experts qui proviennent notamment de l'Assemblée des Premières Nations ont élaboré une stratégie contre la tuberculose, laquelle servira à orienter les programmes de prévention et de contrôle de la tuberculose destinés aux collectivités des Premières Nations sur une réserve (6).

Les nouvelles technologies et approches qui voient le jour à l'échelle mondiale en matière de prévention, de diagnostic et de traitement de la tuberculose pourraient également être prometteuses dans les milieux particuliers où vivent les Autochtones du Canada. Les travaux réalisés par Nunavut Tunngavik Incorporated (NTI), le gouvernement du Nunavut et l'Institut de recherche de l'Hôpital d'Ottawa dans le but de sensibiliser les collectivités à la tuberculose par l'entremise d'une campagne de dépistage à domicile ont récemment fait l'objet d'une publication. Ces travaux pourraient contribuer au succès des mesures de contrôle de la tuberculose chez les collectivités autochtones et mettent en lumière l'efficacité de la recherche communautaire participative auprès des Autochtones (7). La méthode diagnostique Xpert ${ }^{\circledR}$ MTB/RIF, qui est un test de polymérase en chaîne automatisé et niché dans une cartouche (Cepheid, Sunnyvale, Californie) pour la détection rapide de la tuberculose causée par Mycobacterium tuberculosis et de la résistance à la rifampicine (8), est très prometteuse pour la détection précoce de la tuberculose dans les régions éloignées où il n’y a aucun laboratoire spécialisé en tuberculose et où le fardeau de la maladie est souvent parmi les plus élevés au Canada. La méthode de séquençage à haut débit du génome, un outil qui permet de séquencer tous les gènes associés à la tuberculose, offre une résolution beaucoup plus élevée que les méthodes actuelles et semble très prometteuse pour améliorer les connaissances sur la dynamique de transmission de la maladie chez les Autochtones du Canada $(9,10)$. D'autres études portant sur ce sujet sont nécessaires pour caractériser avec plus de précision la dynamique de transmission de la maladie dans cette population. Parmi les autres technologies, il a été démontré que le test de libération d'interféron gamma (TLIG) pour le diagnostic des infections tuberculeuses latentes, une analyse de sang qui n'est généralement pas compromise par le vaccin bacille de Calmette-Guérin (BCG), pouvait être utilisé dans l'Arctique $(3,11)$. Des données récentes d'un vaste essai comparatif randomisé portant sur un traitement significativement plus court de l'infection tuberculeuse latente au moyen de la rifapentine et de l'isoniazide (INH) administrées une fois par semaine pendant 12 semaines ont montré que ce traitement était aussi efficace qu'un traitement de 9 mois par l'INH (la norme actuelle), ce qui est également très prometteur (12). Par ailleurs, ces résultats ont récemment été observés chez les enfants (13).

En attendant la découverte d'un nouveau vaccin efficace contre la tuberculose, nous devons trouver de nouvelles façons de prévenir la maladie chez ceux qui ont une infection latente et de mettre un frein à la transmission de la maladie par ceux qui sont atteints de la tuberculose active. De plus, la découverte de méthodes novatrices visant à atténuer la distribution inégale des déterminants sociaux de la santé qui favorisent la tuberculose chez de nombreuses populations autochtones du Canada est et continuera d'être d'une importance primordiale pour combattre la maladie. Malgré le fait que les taux de tuberculose sont demeurés stables au cours des dernières années au Canada, le combat contre cette maladie n'est pas gagné, la bataille se poursuit donc. Le renouvellement des investissements en recherche, dans l'élaboration de programmes et dans les méthodes d'évaluation qui reposent sur des indicateurs de rendement est indispensable avant d'envisager l'élimination de cette maladie une fois pour toutes chez les Autochtones nés au Canada.

\section{Conflit d'intérêts}

\section{Aucun}

\section{Financement}

Aucun 


\section{Références}

(1) Gallant V, McGuire M, Ogunnaike-Cooke S. A summary of tuberculosis in Canada, 2013. CCDR. 2015 Mar 19;41S2:2-8. (Disponible en français: Résumé des cas de tuberculose au Canada en 2013. RMTC. 2015 19 mars;41 S-2 :2-7).

(2) Long R, Hoeppner V, Orr P, Ainslie M, King M, Abonyi S, et al. Marked disparity in the epidemiology of tuberculosis among Aboriginal peoples on the Canadian prairies: The challenges and opportunities. Canadian Respiratory Journal. 2013;20(4):223-30. Epub 2013/05/30.

(3) Public Health Agency of Canada, Canadian Lung Association, Canadian Thoracic Society. Canadian Tuberculosis Standards. 7th ed. Menzies R, Wong T, editors. Ottawa, ON: Minister of Health; 2013. (Disponible en français: http://www.lignesdirectricesrespiratoires.ca/node/350).

(4) Alvarez GG, Orr, P, Wobeser, WL, Cook, V, Long, R. Tuberculosis prevention and care in First Nations, Inuit and Metis peoples. Canadian Respiratory Journal. 2013;29(Suppl ):44A.

(5) Inuit Tapiriit Kanatami (ITK). Inuit-Specific Tuberculosis (TB) Strategy. Ottawa, ON: ITK; 2013 Mar [cited 2015 Feb]. https://www.itk.ca/publication/inuit-specific-tuberculosis-strategy

(6) Health Canada. Health Canada's Strategy Against Tuberculosis for First Nations On-Reserve. Ottawa, ON: Health Canada; 2012 [cited 2015 Feb].

http://publications.gc.ca/collections/collection_2012/sc-hc/H34-245-2012-eng.pdf.

(Disponible en français: http://www.hc-sc.gc.ca/fniah-spnia/pubs/diseases-maladies/_tuberculos/tuberculos-strateg/factfiche-fra.php).

(7) Alvarez GG, VanDyk DD, Aaron SD, Cameron DW, Davies N, Stephen N, et al. TAIMA (Stop) TB: The impact of a multifaceted TB awareness and door-to-door campaign in residential areas of high risk for TB in Iqaluit, Nunavut. PLoS One. 2014;9(7):e100975. Epub 2014/07/18.

(8) Steingart KR, Schiller I, Horne DJ, Pai M, Boehme CC, Dendukuri N. Xpert(R) MTB/RIF assay for pulmonary tuberculosis and rifampicin resistance in adults. The Cochrane Database of Systematic Reviews. 2014;1:CD009593. Epub 2014/01/23.

(9) Lee RS, Radomski N, Proulx JF, Manry J, Mclntosh F, Desjardins F, et al. Reemergence and amplification of tuberculosis in the Canadian Arctic. The Journal of Infectious Diseases. 2015. Epub 2015/01/13.

(10) Gardy JL, Johnston JC, Ho Sui SJ, Cook VJ, Shah L, Brodkin E, et al. Whole-genome sequencing and social-network analysis of a tuberculosis outbreak. N Engl J Med. 2011;364(8):730-9. Epub 2011/02/25.

(11) Alvarez GG, VanDyk DD, Davies N, Aaron SD, Cameron DW, Desjardins M, et al. The feasibility of the interferon gamma release assay and predictors of discordance with the tuberculin skin test for the diagnosis of latent tuberculosis infection in a remote Aboriginal community. PLoS One. 2014;9(11):e111986. Epub 2014/11/11.

(12) Sterling TR, Villarino ME, Borisov AS, Shang N, Gordin F, Bliven-Sizemore E, et al. Three months of rifapentine and isoniazid for latent tuberculosis infection. N Engl J Med. 2011;365(23):2155-66. Epub 2011/12/14.

(13) Villarino ME, Scott NA, Weis SE, Weiner M, Conde MB, Jones B, et al. Treatment for preventing tuberculosis in children and adolescents: A randomized clinical trial of a 3-month, 12-dose regimen of a combination of rifapentine and isoniazid. JAMA Pediatrics. 2015. Epub 2015/01/13. 